\title{
Serum HER2 levels predict treatment efficacy and prognosis in patients with HER2-positive breast cancer undergoing neoadjuvant treatment
}

\author{
Wen-Jia Zuo ${ }^{1,2 \#}$, Min He ${ }^{1,2 \#}$, Hui Zheng ${ }^{3 \#}$, Yin Liu ${ }^{1,2 \#}$, Xi-Yu Liu ${ }^{1,2}$, Yi-Zhou Jiang ${ }^{1,2}$, Zhong-Hua Wang ${ }^{1,2}$, \\ Ren-Quan $\mathrm{Lu}^{2,3}$, Zhi-Ming Shao ${ }^{1,2}$ \\ ${ }^{1}$ Department of Breast Surgery, Fudan University Shanghai Cancer Center, Shanghai, China; ${ }^{2}$ Department of Oncology, Shanghai Medical College, \\ Fudan University, Shanghai, China; ${ }^{3}$ Department of Clinical Laboratory, Fudan University Shanghai Cancer Center, Shanghai, China \\ Contributions: (I) Conception and design: ZM Shao, RQ Lu; (II) Administrative support: ZM Shao, RQ Lu, ZH Wang; (III) Provision of study \\ materials or patients: All authors; (IV) Collection and assembly of data: WJ Zuo, M He, H Zheng, L Yin; (V) Data analysis and interpretation: WJ \\ Zuo, M He, H Zheng, L Yin; (VI) Manuscript writing: All authors; (VII) Final approval of manuscript: All authors. \\ \#These authors contributed equally to this work. \\ Correspondence to: Zhi-Ming Shao. Department of Breast Surgery, Fudan University Shanghai Cancer Center, 270 Dong-An Road, Shanghai 200032, \\ China. Email: zhimin_shao@yeah.net; Ren-Quan Lu. Department of Clinical Laboratory, Fudan University Shanghai Cancer Center, 270 Dong-An \\ Road, Shanghai 200032, China. Email: lurenquan@126.com.
}

Background: Controversy remains regarding the predictive and prognostic value of serum human epidermal growth factor receptor 2 (HER2) in breast cancer. The purpose of this retrospective study was to determine the clinical utility and efficacy of serum HER2 (sHER2) in predicting treatment response and prognosis in patients with HER2-positive breast cancer undergoing neoadjuvant chemotherapy and trastuzumab treatment.

Methods: A total of 309 HER2-positive breast cancer patients diagnosed at Fudan University Shanghai Cancer Center from July 2015 to January 2019 were analyzed. Baseline sHER2 levels were obtained for all patients and sHER2 levels were collected after 2 cycles of treatment in 208 patients. A sHER2 level $\geq 15 \mathrm{ng} / \mathrm{mL}$ was regarded as "high expression" and sHER2 $<15 \mathrm{ng} / \mathrm{mL}$ was regarded as "low expression". Outcome measures of treatment efficacy and prognosis were pathological complete response (pCR) and invasive disease-free survival (iDFS), respectively.

Results: In patients with high baseline sHER2, more were ER-negative $(\mathrm{P}=0.029)$, had larger tumor size $(\mathrm{P}=0.006)$, more advanced clinical stage $(\mathrm{P}=0.002)$, higher Miller-Payne grade $(\mathrm{P}=0.024)$ and higher likelihood of iDFS events $(\mathrm{P}=0.015)$. Patients with high sHER2 levels after 2 cycles of treatment had lower pCR rates $(\mathrm{P}=0.038)$, higher Miller-Payne grade $(\mathrm{P}=0.013)$ and higher likelihood of iDFS events $(\mathrm{P}=0.003)$. Kaplan-Meier analysis showed significant differences in iDFS between patients with high and low sHER2 levels at baseline $(\mathrm{P}=0.019)$ and after 2 cycles of treatment $(\mathrm{P}=0.000)$. Further analyses according to cancer subtypes found baseline sHER2 to be significantly correlated with the iDFS of Luminal B patients $(\mathrm{p}=0.002)$, while sHER2 levels after 2 cycles of treatment was significantly correlated with the iDFS of HER2-enriched patients $(\mathrm{P}=0.000)$. Univariate analysis showed significant association between iDFS and tumor size $(\mathrm{P}=0.026)$, lymph node status $(\mathrm{P}=0.008)$, clinical stage $(\mathrm{P}=0.031)$, baseline sHER2 $(\mathrm{P}=0.024)$, overall tumor response $(\mathrm{P}=0.011)$, pCR $(\mathrm{P}=0.043)$ and Miller-Payne grade $(\mathrm{P}=0.001)$. Multivariate analysis found Miller-Payne grade $(\mathrm{P}=0.037)$ to be significantly associated with iDFS.

Conclusions: Our results demonstrate the clinical value of sHER2 in a population of Chinese breast cancer patients, suggesting that sHER2 levels after 2 cycles of neoadjuvant therapy may be more predictive of treatment outcomes and that the prognostic value of sHER2 may be time point and subtype dependent.

Keywords: Serum human epidermal growth factor receptor 2 (HER2); HER2-positive breast cancer; neoadjuvant chemotherapy; efficacy; prognosis 
Submitted Nov 02, 2020. Accepted for publication Mar 04, 2021.

doi: 10.21037 /gs-20-802

View this article at: http://dx.doi.org/10.21037/gs-20-802

\section{Introduction}

Breast cancer is the most common type of cancer in women. It is a heterogeneous disease which can be classified into four molecular subtypes with unique clinicopathologic characteristics according to the 2013 St. Gallen consensus: Luminal A, Luminal B, HER2-enriched and Basal-like breast cancer (1-3). In clinical application, immunohistochemical staining of estrogen receptor (ER), progesterone receptor $(\mathrm{PgR})$, human epidermal growth factor receptor-2 (HER2) and Ki67 is commonly used to divide patients into four major subtypes, each with a distinct prognosis (3). HER2-positive or HER2-amplified breast cancer accounts for approximately $20-25 \%$ of invasive breast cancer, and is associated with more aggressive disease, higher risk for progression and decreased overall survival $(4,5)$.

Neoadjuvant chemotherapy (NAC) followed by definitive surgery is a standard of care for locally advanced breast cancer and an option for early-stage breast cancer to increase the chance of breast-conserving surgery (6). The use of NAC allows for an early assessment of treatment effect in breast cancer patients and also provides insight into tumor biology and differential responses to treatment. The main goal of NAC is to achieve pathological complete response (pCR), as past studies have shown pCR to be associated with favorable outcomes (7-9), especially in patients with HER2-positive breast cancer and triple negative breast cancer (TNBC).

With numerous neoadjuvant therapy regimens available and more treatments emerging, the prompt and accurate identification of sensitive responders to NAC is crucial. Unfortunately, many of the traditional biomarkers measured prior to chemotherapy lack accuracy, and most efforts focusing on monitoring morphological changes in tumors are only indicative of a later stage response. In recent decades, numerous predictors of treatment efficacy and prognostic factors have been discovered. Studies examining the relationship between Ki67 expression and chemotherapy response have found breast cancer with a high Ki67 expression level to respond better to chemotherapy (10-12). Similarly, tumor-infiltrating lymphocytes (TILs) have also been reported to be associated with improved distant metastases-free survival in HER2-positive early breast cancer, as well as increased rates of pCR with neoadjuvant trastuzumab and chemotherapy $(13,14)$. However, biomarkers obtained through tissue biopsy would be difficult to monitor repeatedly, limiting its clinical utility, and a liquid biopsy seems more practical. Using liquid biopsies to acquire circulating cancer-derived materials such as circulating tumor cells (CTCs) and circulating tumor DNA (ctDNA) has great diagnostic, predictive and prognostic potential, but still requires further research. Meanwhile, tumor markers such as carcinoembryonic antigen (CEA) and cancer antigen 15-3 (CA 15-3) are easily obtained from blood samples and commonly used in monitoring breast cancer patients, particularly in metastatic setting. Though the sensitivity of these markers may be lacking, study have shown that combination of several tumor markers can enhance the sensitivity for detecting metastatic breast cancer and may merit further exploration (15).

The full-length HER2 protein is a $185 \mathrm{kDa}$ transmembrane receptor composed of an internal tyrosine kinase domain, a short transmembrane domain and an extracellular domain (ECD) (16). The ECD of the HER2 protein can be cleaved from the surface by metalloproteases and detected in the peripheral blood as serum HER2 (sHER2) $(17,18)$. Elevated sHER2 levels (>15 ng/mL) have been observed in $3-15 \%$ of primary breast cancer and up to $46 \%$ in metastatic breast cancer (MBC), studies have shown that increased sHER2 levels are usually associated with poor prognosis (19-30). Serum HER2 can be measured serially and may be used to monitor on-treatment response, predict relapse, or provide a real-time assessment of HER2 status at metastatic presentation $(31,32)$.

However, controversy remains regarding the predictive and prognostic value of sHER2 levels in breast cancer, with contradicting results from different studies (19-30,33,34). It is yet unclear as to whether sHER2 levels predict treatment efficacy and prognosis in patients with HER2-positive breast cancer undergoing neoadjuvant treatment. Thus, the purpose of this retrospective study was to investigate the association between serum HER2 and pCR and invasive disease-free survival (iDFS) in HER2-positive breast cancer patients undergoing neoadjuvant chemotherapy and 
trastuzumab treatment.

We present the following article in accordance with the REMARK reporting checklist (available at http://dx.doi. org/10.21037/gs-20-802).

\section{Methods}

\section{Study population}

In this retrospective study, 309 patients with histologically proven HER2-positive early or locally advanced breast cancer who were diagnosed from July 1, 2015 to January 23, 2019 at Fudan University Shanghai Cancer Center (FUSCC) were analyzed. The study was conducted in accordance with the Declaration of Helsinki (as revised in 2013). The study was approved by ethics committee of FUSCC (No. 050432-4-1212B) and individual consent for this retrospective analysis was waived. Clinical data were retrospectively collected from the electronic medical records system.

Eligible patients were women over 18 years old, diagnosed with histologically confirmed HER2-positive breast cancer, who were treated with neoadjuvant chemotherapy and trastuzumab before surgery. Core needle biopsy of primary tumor was conducted for each patient before neoadjuvant treatment. HER2 positivity was defined as immunohistochemistry (IHC) 3+ or fluorescence in situ hybridization (FISH) positive of the primary tumor. Clinicopathologic information including age, menstrual status, primary tumor size (cT, yp T), regional lymph node status (cN, ypN), ER status, PgR status, HER2 status and Ki67 expression was collected. Patients with incomplete clinicopathological information or without sHER2 data were excluded from this study. Patients were assessed at baseline through clinical examination, laboratory tests, ultrasound (including breast, axillary, supraclavicular, abdominal organs and cardiac ultrasound), mammography, chest computed tomography (CT), breast magnetic resonance imaging (MRI), brain CT or MRI, emission computed tomography (ECT) or positron emission tomography/computed tomography (PET/CT). Treatment efficacy was assessed after every 2 cycles of treatment through clinical examination and imaging-based evaluation (including ultrasound, breast MRI and other necessary methods). Tumor lesions and lymph nodes were assessed according to the Response Evaluation Criteria in Solid Tumours (RECIST) guidelines (version 1.1) (35). Patients underwent definitive surgery after completing neoadjuvant chemotherapy and surgical specimens were assessed by pathologists for pathological response.

Detailed information regarding the patients' neoadjuvant treatment, surgical method and adjuvant treatment was also collected. Post-surgery, patients were treated as needed with adjuvant chemotherapy, anti-HER2 therapy, radiotherapy and endocrine therapy according to clinical guidelines. Follow-up visits were scheduled at 3-month intervals from the date of surgery for the first 2 years, then at 6-month intervals from the date of surgery for years 3 to 5 , and then annually afterwards. Follow-up included clinical examination, ultrasound (breast, axillary, supraclavicular and liver) and laboratory tests at each visit, while mammography and CT was recommended annually. Other exams were added if deemed necessary.

In this study, using the clinicopathologic surrogate definitions of intrinsic subtypes of breast cancer (3), patients who were ER-positive, HER2-positive with any Ki67 and PgR was classified as "Luminal B-like (HER2-positive)" and referred to as "Luminal B"; patients who were HER2positive, ER-negative and $\mathrm{PgR}$-negative was classified as "HER2 positive (non-luminal)" and referred to as "HER2enriched".

\section{Study endpoints}

The primary endpoint of this study was iDFS, defined as the time from the date of primary surgery to the date of breast cancer recurrence, distant metastasis, second primary invasive cancer or death. Subjects who had not experienced iDFS events at the time of analysis were censored using the last assessment date. The secondary end point was $\mathrm{pCR}$, defined as the absence of residual invasive cancer in the breast and lymph nodes (ypT0/is, ypN0). In this study, iDFS was used to measure prognosis and pCR was used to measure treatment efficacy.

\section{Serum HER2 detection}

Peripheral blood was used for sHER2 measurement. Baseline sHER2 was obtained from each patient before treatment was started, and in patients who underwent further testing, sHER2 was also obtained after 2 cycles of neoadjuvant therapy.

The concentration of sHER 2 was measured on ADVIA Centaur CP Immunoassay System (Siemens, Germany). The cut-off value of sHER2 level, $15 \mathrm{ng} / \mathrm{mL}$, is used as reference top-limit in clinical application and 
is the recommended cutoff value according to the Food and Drug Administration and various manufacturer's recommendations for breast cancer (36). Our review of past studies has shown that the cut-off value of sHER2 was set at $15 \mathrm{ng} / \mathrm{mL}$ in most studies and a recent meta-analysis reported the same results (37). In addition, in our previous study (38), we found that patients with sHER $2 \geq 15 \mathrm{ng} / \mathrm{mL}$ had lower progression-free survival than those with sHER2 $<15 \mathrm{ng} / \mathrm{mL}$. Therefore, sHER2 $\geq 15 \mathrm{ng} / \mathrm{mL}$ was regarded as "high expression" and sHER2 $<15 \mathrm{ng} / \mathrm{mL}$ was regarded as "low expression" in this study.

\section{Neoadjuvant treatment}

The majority of patients received PCH (paclitaxel $80 \mathrm{mg} / \mathrm{m}^{2}$, day $1,8,15$ plus carboplatin $\mathrm{AUC}=2$, day 1,8 , 15 plus trastuzumab $4 \mathrm{mg} / \mathrm{kg}$ loading dose and $2 \mathrm{mg} / \mathrm{kg}$ maintenance dose, day $1,8,15,22$ every 4 weeks for 4 to 6 cycles) neoadjuvant therapy. Small numbers of patients received other chemotherapy regimens such as: $\mathrm{TCH}$ (docetaxel $75 \mathrm{mg} / \mathrm{m}^{2}$, day 1 plus carboplatin $\mathrm{AUC}=5$, day 1 plus trastuzumab $8 \mathrm{mg} / \mathrm{kg}$ loading dose and $6 \mathrm{mg} / \mathrm{kg}$ maintenance dose, day 1 every 3 weeks for 6 cycles); TH (docetaxel $100 \mathrm{mg} / \mathrm{m}^{2}$, day 1 plus trastuzumab $8 \mathrm{mg} / \mathrm{kg}$ loading dose and $6 \mathrm{mg} / \mathrm{kg}$ maintenance dose, day 1 every 3 weeks for 4 cycles); PCH-ECH (paclitaxel $80 \mathrm{mg} / \mathrm{m}^{2}$, day $1,8,15$ plus carboplatin $\mathrm{AUC}=2$, day $1,8,15$ plus trastuzumab $4 \mathrm{mg} / \mathrm{kg}$ loading dose and $2 \mathrm{mg} / \mathrm{kg}$ maintenance dose, day $1,8,15,22$ every 4 weeks for 4 cycles, followed by epirubicin $90 \mathrm{mg} / \mathrm{m}^{2}$, day 1 plus cyclophosphamide $600 \mathrm{mg} / \mathrm{m}^{2}$, day 1 plus trastuzumab $6 \mathrm{mg} / \mathrm{kg}$, day 1 every 3 weeks for 4 cycles); ddEC-PH (epirubicin $90 \mathrm{mg} / \mathrm{m}^{2}$, day 1 plus cyclophosphamide $600 \mathrm{mg} / \mathrm{m}^{2}$, day 1 every 2 weeks for 4 cycles, followed by paclitaxel $80 \mathrm{mg} / \mathrm{m}^{2}$, day $1,8,15$ plus trastuzumab $4 \mathrm{mg} / \mathrm{kg}$ loading dose and $2 \mathrm{mg} / \mathrm{kg}$ maintenance dose, day 1,8 , 15 every 3 weeks for 4 cycles) and ddEC-TH (epirubicin $90 \mathrm{mg} / \mathrm{m}^{2}$, day 1 plus cyclophosphamide $600 \mathrm{mg} / \mathrm{m}^{2}$, day 1 every 2 weeks for 4 cycles, followed by docetaxel $100 \mathrm{mg} / \mathrm{m}^{2}$, day 1 plus trastuzumab $8 \mathrm{mg} / \mathrm{kg}$ loading dose and $6 \mathrm{mg} / \mathrm{kg}$ maintenance dose, day 1 every 3 weeks for 4 cycles).

\section{Statistical analysis}

Patient clinicopathological variables were summarized for all participants using standard descriptive statistics. Pearson chi square test was performed to compare qualitative variables.
Logistic regression analyses were performed to determine the relationship between pCR and patient clinicopathological variables, Cox regression was used for the univariate and multivariate analysis of iDFS. Variables found to be significant in univariate analysis were included into multivariate analysis. Factors such as clinical primary tumor size $(\mathrm{cT})$, clinical regional lymph node status $(\mathrm{cN})$, overall tumor response and Miller-Payne Grade were not analyzed individually because some groups had very low numbers of patients. Such factors were categorized into larger groups for the univariate and multivariate survival analysis. Hazard ratios were obtained using Cox proportional hazards regression models, with hazard ratio (HR) $>1$ reflecting a shortened iDFS. A two-tailed P value less than 0.05 was considered as statistically significant. Kaplan-Meier plots were produced for survival endpoint. All statistical analysis was performed using SPSS 24.0 software (SPSS, Chicago, IL, USA).

\section{Results}

\section{Patients and disease characteristics}

In total, 309 HER2-positive patients with baseline sHER2 levels were retrospectively analyzed. The patients' baseline sHER2 levels and disease characteristics are summarized in Table 1. The median age of the patients at diagnosis was 50 years, 147 patients $(47.57 \%)$ were $<50$ years of age and 115 patients $(50.16 \%)$ were premenopausal at time of diagnosis. 81 patients (26.21\%) were stage II and 228 patients $(73.79 \%)$ were stage III at diagnosis. 148 patients (47.90\%) were classified as Luminal B subtype, while 161 patients $(52.10 \%)$ were classified as HER2-enriched subtype.

After neoadjuvant treatment, the 268 patients $(86.73 \%)$ underwent mastectomy, while 41 patients (13.27\%) underwent breast conserving surgery.

\section{Correlation between sHER2 levels and clinicopathological parameters}

The comparison between high baseline sHER2 and low baseline sHER2 patients regarding clinicopathological parameters is listed in Table 2. High sHER2 levels at baseline was associated with tumor size $(\mathrm{P}=0.006)$, clinical stage $(\mathrm{P}=0.002)$, ER status $(\mathrm{P}=0.029)$, breast cancer subtype ( $\mathrm{P}=0.029)$, mastectomy $(\mathrm{P}=0.003)$, Miller-Payne grade $(\mathrm{P}=0.024)$.

Out of the overall 309 patients, 208 patients were further 
Table 1 Patient Characteristics

\begin{tabular}{|c|c|c|}
\hline Patient Characteristics & $N=309$ & Percentage (\%) \\
\hline \multicolumn{3}{|l|}{ Age group } \\
\hline$<50$ years & 147 & 47.57 \\
\hline$\geq 50$ years & 162 & 52.43 \\
\hline \multicolumn{3}{|l|}{ Menstrual status } \\
\hline Premenopausal & 155 & 50.16 \\
\hline Postmenopausal & 154 & 49.84 \\
\hline \multicolumn{3}{|l|}{ cT } \\
\hline $\mathrm{T} 1$ & 12 & 3.88 \\
\hline $\mathrm{T} 2$ & 108 & 34.95 \\
\hline T3 & 122 & 39.48 \\
\hline $\mathrm{T} 4$ & 67 & 21.68 \\
\hline \multicolumn{3}{|l|}{$\mathrm{cN}$} \\
\hline No & 21 & 6.80 \\
\hline $\mathrm{N} 1$ & 155 & 50.16 \\
\hline $\mathrm{N} 2$ & 88 & 28.48 \\
\hline N3 & 45 & 14.56 \\
\hline \multicolumn{3}{|l|}{ Clinical stage } \\
\hline II & 81 & 26.21 \\
\hline III & 228 & 73.79 \\
\hline \multicolumn{3}{|l|}{ ER status } \\
\hline Negative & 161 & 47.90 \\
\hline Positive & 148 & 52.10 \\
\hline \multicolumn{3}{|l|}{ PgR status } \\
\hline Negative & 218 & 29.45 \\
\hline Positive & 91 & 70.55 \\
\hline \multicolumn{3}{|l|}{ Subtype } \\
\hline Luminal B & 148 & 47.90 \\
\hline HER2-enriched & 161 & 52.10 \\
\hline \multicolumn{3}{|l|}{ Overall tumor response } \\
\hline $\mathrm{CR}$ & 24 & 7.77 \\
\hline PR & 279 & 90.29 \\
\hline SD & 5 & 1.62 \\
\hline PD & 1 & 0.32 \\
\hline \multicolumn{3}{|l|}{$\mathrm{pCR}$} \\
\hline Yes & 134 & 43.37 \\
\hline No & 175 & 56.63 \\
\hline
\end{tabular}

Table 1 (continued)
Table 1 (continued)

\begin{tabular}{lcc}
\hline Patient Characteristics & $\mathrm{N}=309$ & Percentage (\%) \\
\hline Miller-Payne Grade & 13 & 4.21 \\
1 & 12 & 3.88 \\
2 & 53 & 17.15 \\
3 & 60 & 19.42 \\
4 & 139 & 44.98 \\
5 & 32 & 10.36 \\
NA & & \\
iDFS events & 281 & 90.94 \\
No & 28 & 9.06 \\
Yes & & \\
Surgery & 41 & 13.27 \\
Breast conserving & 268 & 86.73 \\
Mastectomy &
\end{tabular}

According to AJCC Cancer Staging Manual, Eighth Edition (2017): cT, clinical primary tumor size; cN, clinical regional lymph nodes. ER, estrogen receptor; PgR, progesterone receptor; HER2, human epidermal growth factor receptor 2; CR, complete response; PR, partial response; SD, stable disease; PD, progressive disease; $p C R$, pathological complete response; NA, not available; iDFS, invasive disease-free survival.

tested for sHER2 levels after 2 cycles of neoadjuvant treatment. When divided into high and low sHER2 cohorts according to sHER2 levels, high sHER2 expression after 2 cycles of neoadjuvant therapy was found to be associated with pCR $(\mathrm{P}=0.038)$ and Miller-Payne grade $(\mathrm{P}=0.013)$. No statistical relationship was observed between the sHER2 levels after 2 cycles of neoadjuvant therapy and other variables including age, menstrual status, tumor size, nodal status, ER status, PgR status, breast cancer subtype, overall tumor response nor surgical method.

\section{The predictive value of sHER2 in regard to $p C R$}

In this study, postsurgical pathology revealed that 134 patients $(43.37 \%)$ achieved pCR, while 175 patients (56.63\%) were non-pCR and had residual disease. As shown in Table2, high sHER2 levels at baseline was not associated with pCR $(\mathrm{P}=0.159)$, but high sHER2 levels after 2 cycles of neoadjuvant treatment was found to be associated with a lower pCR rate $(\mathrm{P}=0.038)$. Logistic regression confirmed pCR to be significantly correlated with sHER2 levels after 
Table 2 Clinicopathological variables and serum HER2 levels at baseline and after 2 cycles of treatment in the study cohort

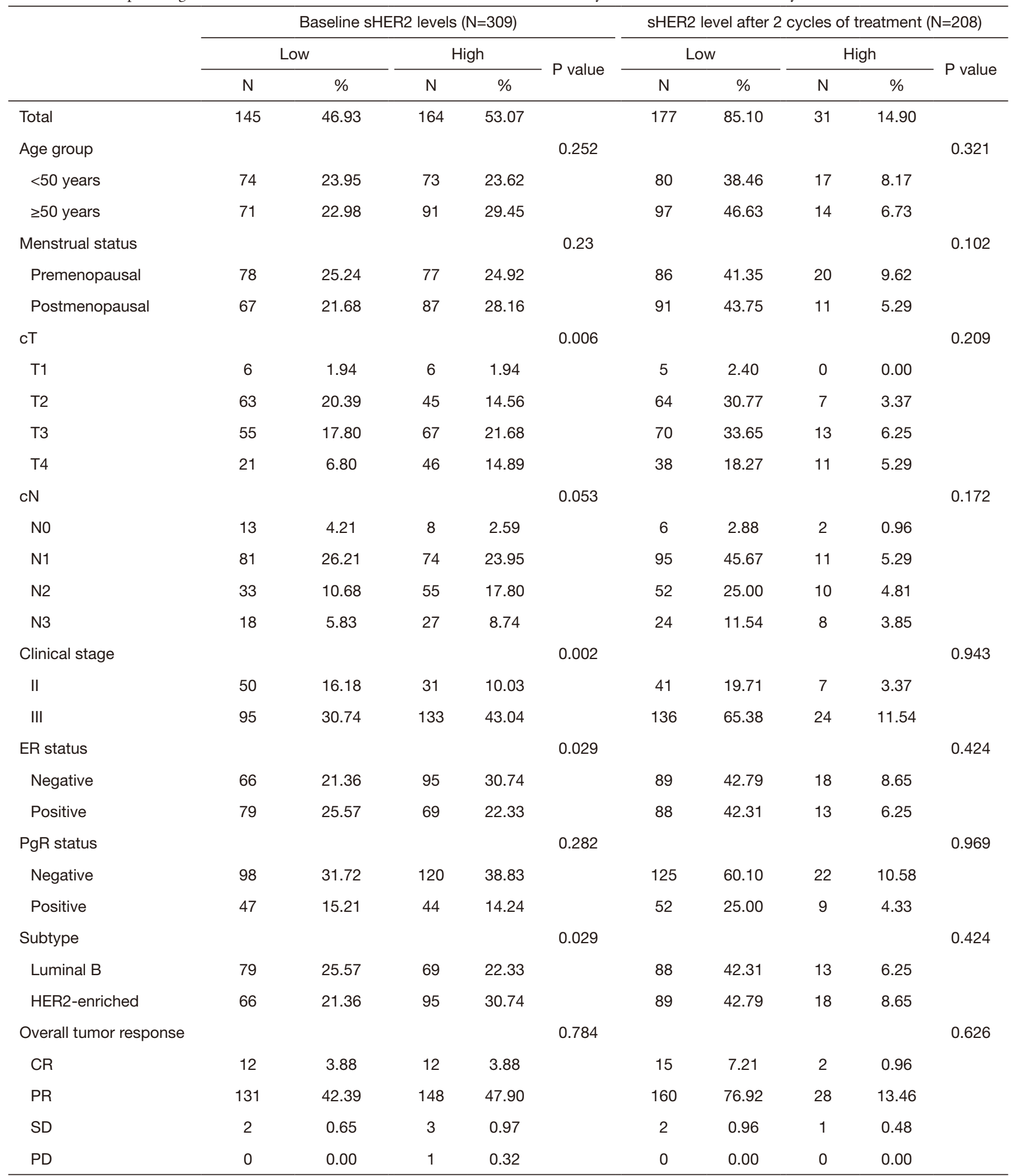

Table 2 (continued) 
Table 2 (continued)

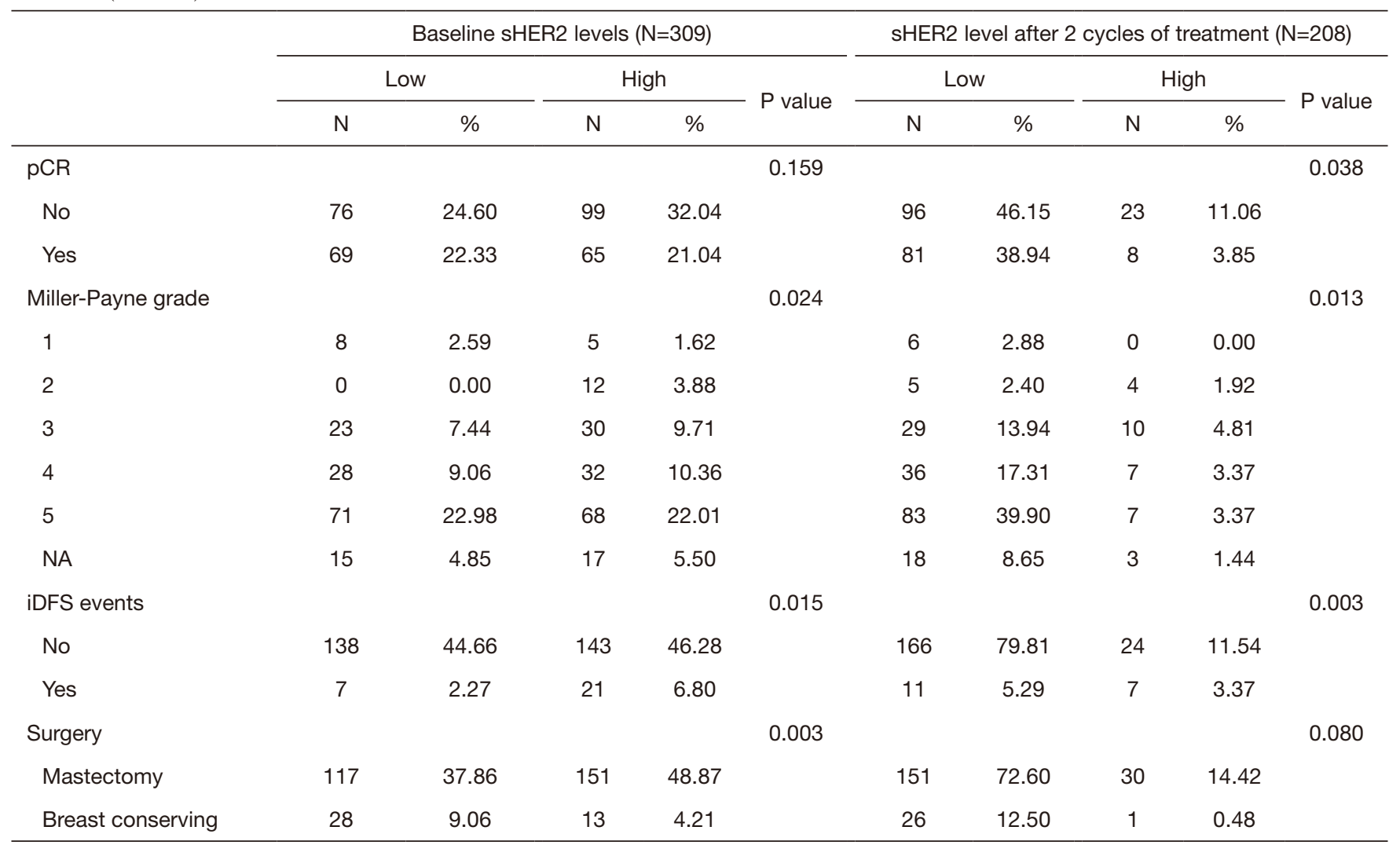

According to AJCC Cancer Staging Manual, Eighth Edition (2017): cT, clinical primary tumor size; cN, clinical regional lymph nodes. sHER2, serum human epidermal growth factor receptor 2; ER, estrogen receptor; PgR, progesterone receptor; HER2, human epidermal growth factor receptor 2; CR, complete response; PR, partial response; SD, stable disease; PD, progressive disease; pCR, pathological complete response; NA, not available; iDFS, invasive disease-free survival.

2 cycles of neoadjuvant therapy $(\mathrm{P}=0.043)$, but not with baseline sHER2 levels $(\mathrm{P}=0.160)$. Interestingly, when we further analyzed the predictive value of sHER2 levels in different subtypes, we found that only the sHER 2 after 2 cycles of neoadjuvant treatment in HER2-enriched breast cancer was statistically significant $(\mathrm{P}=0.005)$. Details are listed in Table 3.

Previous studies by Witzel et al. had reported that a decrease in sHER2 levels $(>20 \%)$ during treatment of HER2-positive breast cancer was significantly associated with higher pCR rates $(25,26)$ in patients undergoing neoadjuvant treatment and longer progression free survival (PFS) in metastatic breast cancer patients (39). Study by Mazouni et al. suggested decrease in sHER2 (>9\%) to be predictive of pCR (40). On this basis, we further evaluated the decrease of sHER2 after neoadjuvant treatment and its relations to treatment efficacy and patient prognosis, but no significant association was found between the decrease in
sHER2 levels ( $>20 \%$ and $>9 \%$ ) after 2 cycles compared to baseline and patient $\mathrm{pCR}(\mathrm{P}=0.871$ and 0.967 , respectively). Receiver operating characteristic (ROC) curve analysis was also used to search for a suitable cut-off level for decrease in sHER2 levels to predict pCR but did not yield satisfactory results.

\section{The prognostic value of sHER2 in regard to iDFS}

The median follow-up duration was 23.3 months. At the time of analysis, 28 patients $(9.06 \%)$ had experienced iDFS event, the overall median iDFS was 20.8 months.

Kaplan-Meier analysis of the overall patients showed significant differences in iDFS between the low baseline sHER2 and high baseline sHER2 cohorts (log-rank $=5.524$, $\mathrm{P}=0.019$ ) (Figure 1A). Interestingly, when we further evaluated the patients according to breast cancer subtypes, Luminal B patients showed significant difference in iDFS 
Table 3 Logistic Regression results of pCR in relations to sHER2 levels

\begin{tabular}{|c|c|c|c|}
\hline Subtype & $\mathrm{HR}$ & $95 \% \mathrm{Cl}$ & $P$ value \\
\hline \multicolumn{4}{|c|}{ Baseline sHER2 (N=309) } \\
\hline Overall & 0.723 & $0.460-1.136$ & 0.160 \\
\hline Luminal B & 0.530 & $0.265-1.058$ & 0.072 \\
\hline HER2-enriched & 0.782 & $0.417-1.469$ & 0.445 \\
\hline Overall & 0.412 & $0.175-0.971$ & 0.043 \\
\hline Luminal B & 1.004 & $0.284-3.547$ & 0.995 \\
\hline HER2-enriched & 0.185 & $0.056-0.609$ & 0.005 \\
\hline
\end{tabular}

pCR, pathological complete response; sHER2, serum human epidermal growth factor receptor 2; HR, hazard ratio; $\mathrm{Cl}$, confidence interval; HER2, human epidermal growth factor receptor 2.

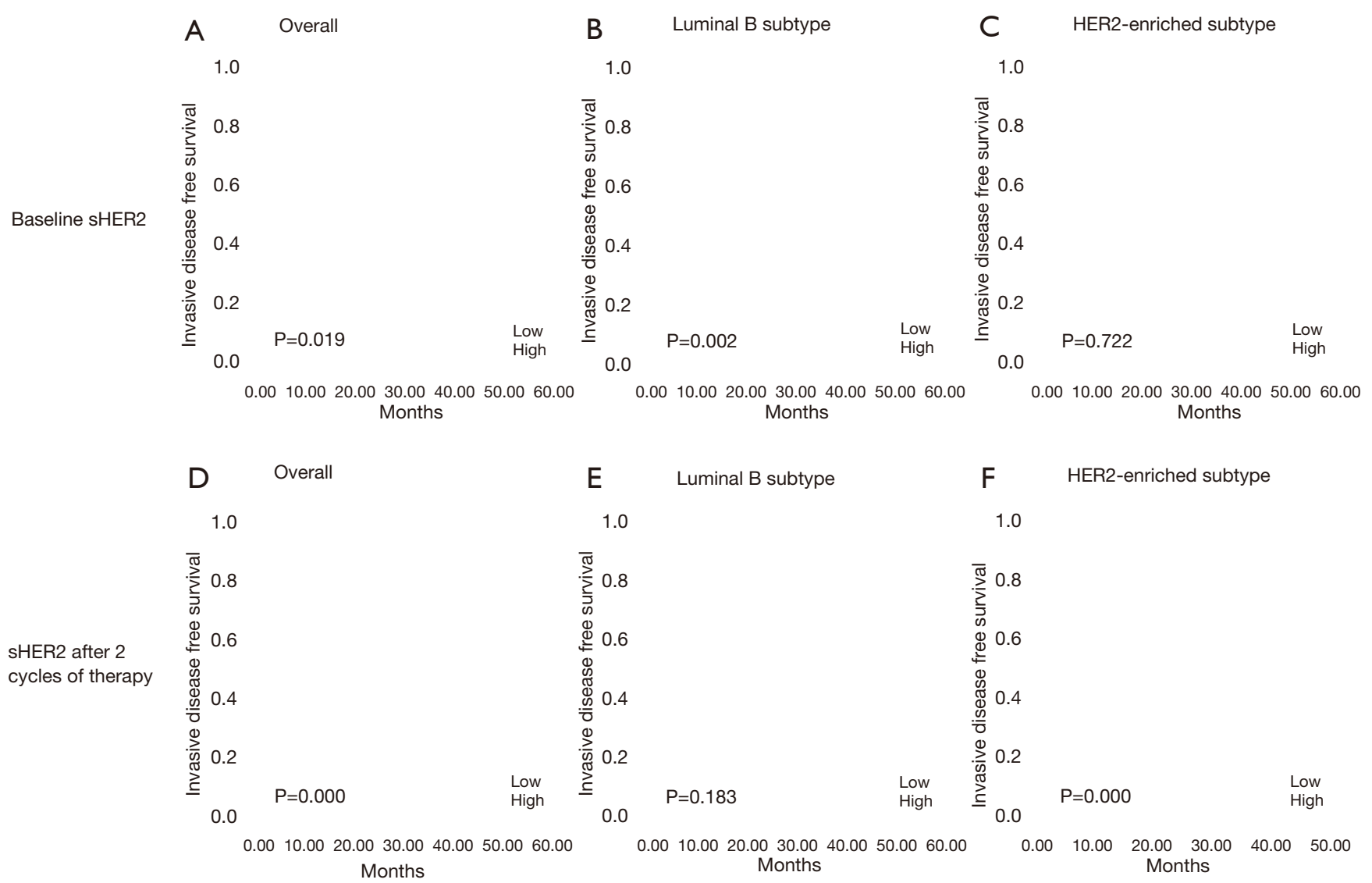

Figure 1 Invasive disease-free survival (iDFS) according to serum HER2 (sHER2) levels. High: sHER2 $\geq 15$ ng/mL; Low: sHER2 $<15 \mathrm{ng} / \mathrm{mL}$. Baseline sHER2 levels and iDFS in (A) overall patients; (B) Luminal B subtype patients; (C) HER2-enriched subtype patients; sHER2 levels after 2 cycles of neoadjuvant treatment and iDFS in (D) overall patients; (E) Luminal B subtype patients; (F) HER2-enriched subtype patients. 
$(\log$-rank $=9.506, \mathrm{P}=0.002)$ (Figure $1 B)$, while there was no significant difference between the two cohorts in HER2enriched patients (log-rank $=0.127, \mathrm{P}=0.722$ ) (Figure 1C). In addition, in our analysis of the 208 patients with subsequent data regarding sHER2 levels after 2 cycles of treatment, we observed a significant difference in iDFS between the low sHER2 and high sHER2 cohorts (log-rank $=14.353$, $\mathrm{P}=0.000$ ) (Figure 1D). Evaluation according to subtypes revealed that there was no significant difference in iDFS between the two cohorts in Luminal B patients (logrank $=1.770, \mathrm{P}=0.183$ ) (Figure $1 E$ ), but found significant difference in HER2-enriched patients (log-rank $=13.977$, $\mathrm{P}=0.000$ ) (Figure $1 F$ ). Similarly, we evaluated the decrease of sHER2 after neoadjuvant treatment and its relations to patient prognosis, but no significant association was found between the decrease of sHER2 and patient iDFS $(\mathrm{P}=0.213)$.

In our initial analysis of clinicopathological variables and sHER2 levels (Table 2), high sHER2 levels at baseline and after 2 cycles of neoadjuvant treatment were found to be associated to iDFS events ( $\mathrm{P}=0.015$ and 0.003 , respectively). Cox proportional hazard regression was then used to explore prognostic factors associated with iDFS and our results are shown in Table 4. We observed a significant association between iDFS and tumor size $(\mathrm{P}=0.026)$, lymph node status $(\mathrm{P}=0.008)$, clinical stage $(\mathrm{P}=0.031)$, baseline sHER2 $(\mathrm{P}=0.024)$, overall tumor response $(\mathrm{P}=0.011)$, $\mathrm{pCR}$ $(\mathrm{P}=0.043)$ and Miller-Payne grade $(\mathrm{P}=0.001)$ in univariate analysis. When we further corrected these factors in multivariate analysis, the results revealed that patients with higher Miller-Payne grade experienced significantly longer iDFS than their counterparts (HR $=0.374,95 \% \mathrm{CI}$ : 0.148-0.945, $\mathrm{P}=0.037)$. Baseline sHER2 levels proved to be insignificant in multivariate analysis.

\section{Discussion}

HER2 is an important therapeutic target in breast cancer, and current clinical guidelines strongly advocate the usage of anti-HER2 treatment and chemotherapy in the treatment of HER2-positive patients. Serum HER2 has been proposed as a promising prognostic and predictive biomarker for HER2-positive patients, but evidence regarding sHER2 remains controversial and inconclusive.

In the adjuvant setting, results from North Central Cancer Treatment Group Adjuvant Trial N9831 showed that elevated baseline sHER2 level was a prognostic biomarker associated with shorter disease-free survival (DFS) and a high sHER2 level at recurrence was predictive of shorter survival (30). Similarly, Reix et al. observed that DFS was shorter in patients with elevated sHER2 levels ( $\geq 15 \mathrm{ng} / \mathrm{mL}$ ) compared to those with low sHER2 levels. In addition, an increase in sHER2 was found to be useful in some cases to detect recurrences at an early stage (41). A study by Lee et al. of 436 stage I-III breast cancer of varying subtypes showed that high levels of sHER2 at diagnosis were associated with shorter DFS in hormone receptor+/ HER2 -, hormone receptor+/HER2 + and hormone receptor-/HER2 + patients, suggesting sHER2 to be a useful prognostic factor in operable breast cancer (32). But in said study, only 8 patients out of 268 patients with hormone receptor+/HER2 - tumors had elevated sHER2 levels and the evidence may be insufficient for us to conclude that sHER2 is a useful prognostic factor in hormone receptor+/ HER2- breast cancer.

In MBC, patients with metastases were more likely to have elevated sHER2 levels than patients without metastases, and elevated sHER2 levels were found to be correlated with parameters associated with tumor aggressiveness (41). Multiple studies have suggested that elevated sHER2 and a lack of decline after treatment is associated with poorer survival, and that sHER2 may be a useful surveillance biomarker for detecting early signs of recurrence and to predict the fate of metastases (31,41-46). However, contrary evidence does remain from a pooled analysis of four MBC trials of trastuzumab that found no clear relationship between baseline sHER2 levels and tumor response, no significant relationship between decline in sHER2 levels and tumor, and that disease progression could not be reliably predicted by rising sHER2 levels in the majority of patients (34).

The value of sHER2 in the neoadjuvant setting also remains inconclusive. While some studies have found elevated baseline sHER2 levels to be associated with a better response to trastuzumab-based treatment $(25,47)$, others did not find significant difference in mean baseline sHER2 values between the pCR group and non-PCR group (40). In neoadjuvant trials of large operable or locally advanced HER2-positive breast cancer, results from the GeparQuattro and GeparQuinto trials found that both an elevated sHER2 level $(>15 \mathrm{ng} / \mathrm{mL})$ and a significant reduction of sHER2 between two successive blood draws (a decrease of $>20 \%$ measured before and after NAC) were significantly associated with higher pCR after chemotherapy and anti-HER2 treatment (trastuzumab and lapatinib) $(25,26)$. A small prospective trial by Mazouni et al. of 39 patients observed no significant difference in baseline 
Table 4 Univariate and multivariate survival analysis of factors associated with invasive disease-free survival (N=309)

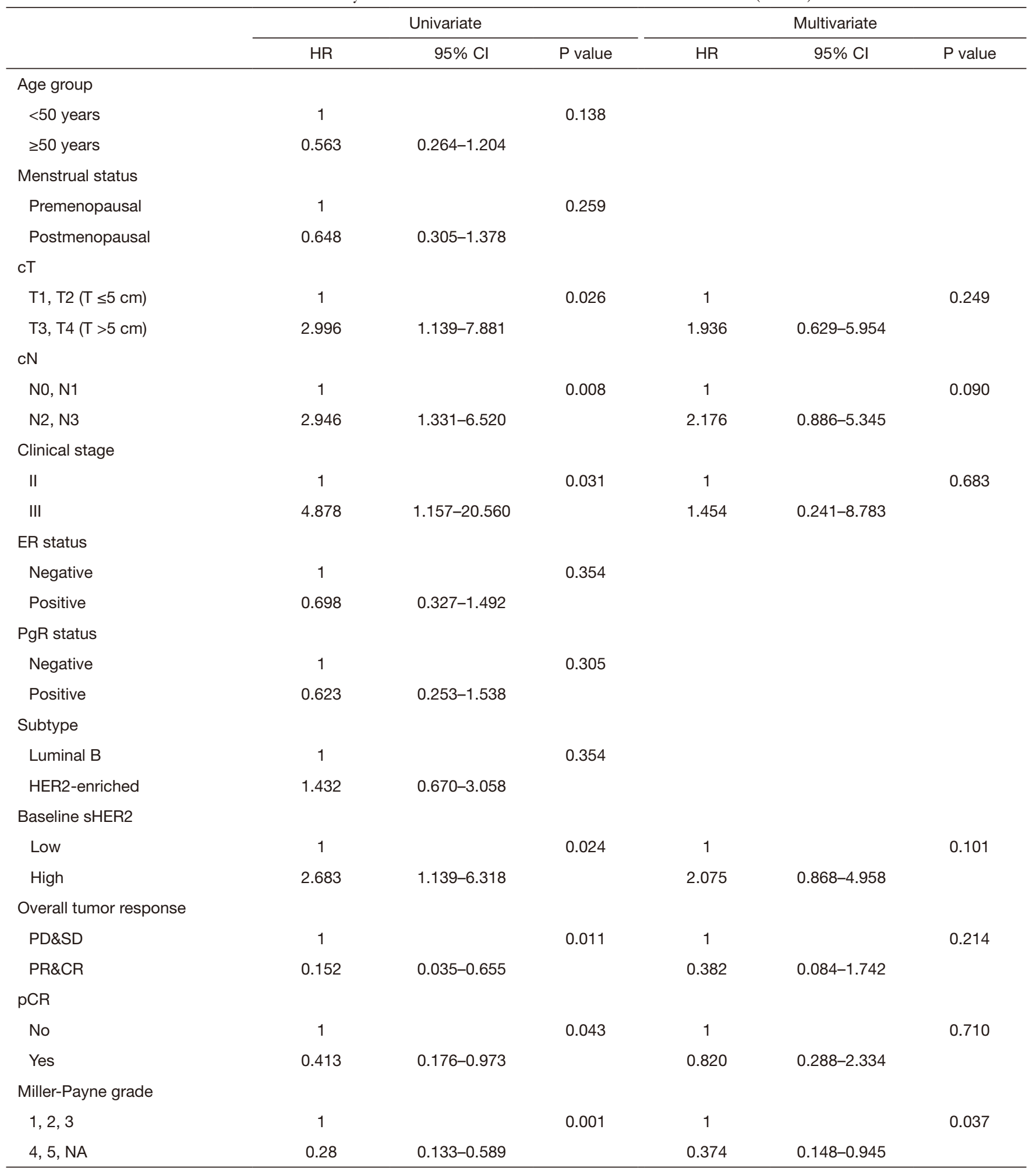

According to AJCC Cancer Staging Manual, Eighth Edition (2017): cT, clinical primary tumor size; cN, clinical regional lymph nodes. HR, hazard ratio; $\mathrm{Cl}$, confidence interval; ER, estrogen receptor; PgR, progesterone receptor; HER2, human epidermal growth factor receptor 2; $\mathrm{CR}$, complete response; PR, partial response; SD, stable disease; PD, progressive disease; pCR, pathological complete response; NA, not available; iDFS, invasive disease-free survival. 
sHER2 levels between patients who achieved pCR and those with residual disease, but reported that a decrease in sHER2 levels early during treatment to be associated with pCR (40).

In this study, we attempted to evaluate the value of sHER2 as a predictive and prognostic factor for HER2positive patients undergoing neoadjuvant treatment. To our knowledge, this study is the largest one to evaluate sHER2 in HER2-positive breast cancer patients undergoing neoadjuvant treatment.

Our results indicate that elevated levels of sHER2 did correlate positively with some clinicopathological parameters related to tumor aggressiveness and poor prognosis such as: larger tumor size, more advanced clinical stage and negative ER status. Surprisingly, factors such as menstrual status and lymph node status were not correlated with sHER2 levels. Linear regression found no correlation between age and sHER2 levels. We can infer that sHER2 levels are not age-dependent, and therefore unrelated to patient menstrual status. We also surmise that the lack of significant correlation between sHER2 and lymph node status may be due to the small number of lymph node negative patients included in this study, as the majority of patients receiving neoadjuvant treatment presented with axillary lymph node involvement at diagnosis.

Contrary to results from previous clinical trials $(25,26,40)$, our study found that neither an elevated baseline sHER2 nor a decrease in sHER2 after treatment were significantly associated with pCR. However, we found high sHER2 levels after 2 cycles of neoadjuvant treatment to be significantly associated with pCR. There are several explanations for the discrepancies of our results. First of all, ethnicity is a possible factor as it may influence baseline levels of sHER2, and Asian patients have been previously reported to have significantly higher sHER2 levels than other ethnicities $(28,48)$. Secondly, the lack of association between decrease in sHER 2 and pCR may be explained by the difference in our time points of obtaining post-treatment sHER2 levels. In the GeparQuattro study, sHER2 was obtained before and after treatment in patients randomized to receive 8 to 12 cycles of neoadjuvant chemotherapy and trastuzumab. Decrease of sHER2 levels (>20\%) after chemotherapy was associated with pCR, but sHER2 levels obtained after the end of chemotherapy showed no difference between pCR and non-pCR patients. Similarly, in the GeparQuinto study, sHER2 was obtained before treatment, after 4 cycles of treatment and after 8 cycles of treatment in patients randomized to receive 8 cycles of neoadjuvant chemotherapy and either trastuzumab or lapatinib antiHER2 treatment. A decline of sHER2 levels (>20\%) after 4 cycles was reported to be associated with pCR but the GeparQuinto study did not report whether sHER2 levels after 4 cycles were associated with pCR. In the study by Mazouni et al., sHER2 levels were obtained at baseline before initial chemotherapy and before each subsequent cycle of treatment to find the threshold value that best predicted pCR. A decrease by $9 \%$ in sHER 2 from week 3 to 6 was found to be statistically significant, while a decrease by $9 \%$ in sHER 2 from baseline to week 6 was also the best threshold value for predicting pCR but was not statistically significant. Finally, the difference in treatment regimens may also be a crucial factor. The majority of patients in our study received taxane based chemotherapy with trastuzumab treatment, while patients of the GeparQuattro study received trastuzumab with epirubicin/cyclophosphamide followed by randomization to either docetaxel alone, docetaxel in combination with capecitabine or docetaxel followed by capecitabine. Meanwhile, patients of the GeparQuinto study received epirubicin/cyclophosphamide followed by docetaxel and were randomly assigned to receive concomitant trastuzumab or lapatinib, and most notably, patients in the study by Mazouni et al. were randomized to receive paclitaxel followed by fluorouracil/epirubicin/ cyclophosphamide chemotherapy alone or in combination with trastuzumab. The differences in chemotherapy and anti-HER2 therapy may be a significant factor in explaining why our results differed from previous studies. Interestingly, as we mentioned above, we found elevated sHER2 levels after 2 cycles of neoadjuvant treatment to be associated with pCR, suggesting that it may be a viable predictor of tumor response in patients undergoing chemotherapy and trastuzumab treatment. Patients undergoing neoadjuvant treatment at FUSCC are routinely evaluated for tumor response through physical examination and imaging examination after every 2 cycles of treatment and sHER2 levels may be a useful biomarker to help predict treatment efficacy and tumor response, especially in HER2-positive patients.

Univariate analysis found iDFS to be associated with commonly accepted variables indicative of poor prognosis such as: tumor size, lymph node status, overall tumor response, pCR and Miller-Payne grade after treatment. Elevated sHER2 both at baseline and after 2 cycles of treatment was observed to be significantly associated with shorter iDFS, and our results are in accordance with previous reports that high levels of sHER2 is associated with 
worse prognosis $(41,48,49)$. However, a novel finding in our study is that elevated sHER2 levels at different timepoints during neoadjuvant treatment may have unique prognostic value for different subtypes of breast cancer. High sHER2 levels at baseline was associated with significant iDFS differences in Luminal B patients, while high sHER2 levels after 2 cycles of treatment was associated with significant iDFS differences in HER2-enriched patients. Not only does this offer us more precise guidance in employing sHER2 as a prognostic factor, our results suggest that when assessing the prognostic value of sHER2, we should further divide HER2-positive patients into Luminal B and HER2enriched subtypes based upon their ER and PgR status, for this may yield more accurate results.

Inevitably, our study has its limitations. First, our study was performed using retrospective data rather than prospective cohorts, and sampling bias may have been introduced. Second, patients in this study were treated trastuzumab concomitantly with chemotherapy. While the majority of patients received 6 cycles of $\mathrm{PCH}$ neoadjuvant treatment, a small percentage of patients underwent other chemotherapy regimens and underwent surgery after 4-8 cycles of treatment depending on their chemotherapy regimen. Though the majority of HER2-positive patients respond well to chemotherapy and anti-HER2 treatment, the different regimens and cycles of chemotherapy may affect pCR rates. Third, current clinical guidelines recommend trastuzumab and pertuzumab in addition to taxane based chemotherapy for HER2 positive patients receiving neoadjuvant chemotherapy, and whether our results are applicable to patients undergoing dual antiHER2 treatment remains to be explored. Finally, although our study included a substantial sample size, with a baseline sHER2 from 309 patients and subsequent sHER2 data from 208 patients, our results are from a single center and only 28 iDFS events were observed in overall patients. Thus, our results should be interpreted with caution, and further study in prospective patients will be needed to validate the predictive and prognostic value of sHER2 in neoadjuvant, adjuvant and metastatic settings, especially within the context of newer anti-HER2 therapies, such as lapatinib, pyrotinib, pertuzumab and trastuzumab-emtansine.

Nevertheless, our study offers new insights into the predictive and prognostic value of sHER2 levels in neoadjuvant treatment of HER2-positive breast cancer. The development of new technology and novel drugs has powered the advances of individualized treatment of breast cancer. The phase Ib/II Fudan University Shanghai Cancer Center TNBC umbrella (FUTURE) trial has shown promising results in the precision treatment of refractory metastatic patients (50), and with our plans to expand targeted treatment to the neoadjuvant setting, the results from this study offers clues in how to identify patients who may benefit from novel targeted therapies.

\section{Conclusions}

Our study brings evidences to support the use of sHER2 in the neoadjuvant treatment of HER2-positive breast cancer patients. Our results demonstrate the predictive and prognostic value of sHER2 in a population of Chinese breast cancer patients, suggesting that sHER2 levels after 2 cycles may be more predictive of treatment efficacy and that the prognostic value of sHER2 may be time point and subtype dependent. Therefore, we would advocate the measurement of sHER2 levels after 2 cycles of treatment in addition to baseline, and we also suggest categorizing HER2-positive into Luminal B and HER2-enriched subtypes when evaluating sHER2. As a high sHER2 level predicts worse prognosis, sHER2 may be used to identify and further stratify high risk patients who may benefit from intensive treatment and newer targeted therapies in future studies.

\section{Acknowledgments}

The authors are grateful to all patients who participated in the study, we acknowledge Miao Mo for assistance in data analysis.

Funding: This work was supported by the National Natural Science Foundation of China (81802638), the Shanghai Committee of Science and Technology Funds (18ZR1407500) and the Fudan University Shanghai Cancer Center Institute Research Fund (YJ201805).

\section{Footnote}

Reporting Checklist: The authors have completed the REMARK checklist. Available at http://dx.doi. org/10.21037/gs-20-802

Data Sharing Statement: Available at http://dx.doi. org/10.21037/gs-20-802

Conflicts of Interest: All authors have completed the ICMJE 
uniform disclosure form (available at http://dx.doi. org/10.21037/gs-20-802). Dr. MH reports grants from National Natural Science Foundation of China, grants from Shanghai Committee of Science and Technology Funds, during the conduct of the study. The other authors have no conflict of interests to declare.

Ethical Statement: The authors are accountable for all aspects of the work in ensuring that questions related to the accuracy or integrity of any part of the work are appropriately investigated and resolved. The study was conducted in accordance with the Declaration of Helsinki (as revised in 2013). The study was approved by ethics committee of Fudan University Shanghai Cancer Center (NO. 050432-4-1212B) and individual consent for this retrospective analysis was waived.

Open Access Statement: This is an Open Access article distributed in accordance with the Creative Commons Attribution-NonCommercial-NoDerivs 4.0 International License (CC BY-NC-ND 4.0), which permits the noncommercial replication and distribution of the article with the strict proviso that no changes or edits are made and the original work is properly cited (including links to both the formal publication through the relevant DOI and the license). See: https://creativecommons.org/licenses/by-nc-nd/4.0/.

\section{References}

1. Perou CM, Sorlie T, Eisen MB, et al. Molecular portraits of human breast tumours. Nature 2000;406:747-52.

2. Parker JS, Mullins M, Cheang MC, et al. Supervised risk predictor of breast cancer based on intrinsic subtypes. $\mathrm{J}$ Clin Oncol 2009;27:1160-7.

3. Goldhirsch A, Winer EP, Coates AS, et al. Personalizing the treatment of women with early breast cancer: highlights of the St Gallen International Expert Consensus on the Primary Therapy of Early Breast Cancer 2013. Ann Oncol 2013;24:2206-23.

4. Slamon DJ, Clark GM, Wong SG, et al. Human breast cancer: correlation of relapse and survival with amplification of the HER-2/neu oncogene. Science 1987;235:177-82.

5. Slamon DJ, Godolphin W, Jones LA, et al. Studies of the HER-2/neu proto-oncogene in human breast and ovarian cancer. Science 1989;244:707-12.

6. Mieog JS, van der Hage JA, van de Velde CJ. Preoperative chemotherapy for women with operable breast cancer.
Cochrane Database Syst Rev 2007;2007:CD005002.

7. Rouzier R, Pusztai L, Delaloge S, et al. Nomograms to predict pathologic complete response and metastasis-free survival after preoperative chemotherapy for breast cancer. J Clin Oncol 2005;23:8331-9.

8. Prowell TM, Pazdur R. Pathological complete response and accelerated drug approval in early breast cancer. $\mathrm{N}$ Engl J Med 2012;366:2438-41.

9. Liu Q, Wang C, Li P, et al. The Role of (18)F-FDG PET/CT and MRI in Assessing Pathological Complete Response to Neoadjuvant Chemotherapy in Patients with Breast Cancer: A Systematic Review and Meta-Analysis. Biomed Res Int 2016;2016:3746232.

10. Kim KI, Lee KH, Kim TR, et al. Ki-67 as a predictor of response to neoadjuvant chemotherapy in breast cancer patients. J Breast Cancer 2014;17:40-6.

11. Urruticoechea A, Smith IE, Dowsett M. Proliferation marker Ki-67 in early breast cancer. J Clin Oncol 2005;23:7212-20.

12. Fasching PA, Heusinger K, Haeberle L, et al. Ki67, chemotherapy response, and prognosis in breast cancer patients receiving neoadjuvant treatment. BMC Cancer 2011;11:486.

13. Denkert C, Loibl S, Noske A, et al. Tumor-associated lymphocytes as an independent predictor of response to neoadjuvant chemotherapy in breast cancer. J Clin Oncol 2010;28:105-13.

14. Loi S, Michiels S, Salgado R, et al. Tumor infiltrating lymphocytes are prognostic in triple negative breast cancer and predictive for trastuzumab benefit in early breast cancer: results from the FinHER trial. Ann Oncol 2014;25:1544-50.

15. Pedersen AC, Sorensen PD, Jacobsen EH, et al. Sensitivity of CA 15-3, CEA and serum HER2 in the early detection of recurrence of breast cancer. Clin Chem Lab Med 2013;51:1511-9.

16. Wang Z. ErbB Receptors and Cancer. Methods Mol Biol 2017;1652:3-35.

17. Codony-Servat J, Albanell J, Lopez-Talavera JC, et al. Cleavage of the HER2 ectodomain is a pervanadateactivable process that is inhibited by the tissue inhibitor of metalloproteases- 1 in breast cancer cells. Cancer Res 1999;59:1196-201.

18. Tsé C, Gauchez AS, Jacot W, et al. HER2 shedding and serum HER2 extracellular domain: biology and clinical utility in breast cancer. Cancer Treat Rev 2012;38:133-42.

19. Narita T, Funahashi H, Satoh Y, et al. C-erbB-2 protein in the sera of breast cancer patients. Breast Cancer Res Treat 
1992;24:97-102.

20. Molina R, Jo J, Filella X, et al. c-erbB-2 oncoprotein, CEA, and CA 15.3 in patients with breast cancer: prognostic value. Breast Cancer Res Treat 1998;51:109-19.

21. Fehm T, Maimonis P, Weitz S, et al. Influence of circulating c-erbB-2 serum protein on response to adjuvant chemotherapy in node-positive breast cancer patients. Breast Cancer Res Treat 1997;43:87-95.

22. Molina MA, Saez R, Ramsey EE, et al. NH(2)-terminal truncated HER-2 protein but not full-length receptor is associated with nodal metastasis in human breast cancer. Clin Cancer Res 2002;8:347-53.

23. Pichon MF, Hacene K, Guepratte S, et al. Serum HER-2 extracellular domain (ECD) before the first metastasis in 128 breast cancer patients. Clin Lab 2004;50:163-70.

24. Ludovini V, Gori S, Colozza M, et al. Evaluation of serum HER2 extracellular domain in early breast cancer patients: correlation with clinicopathological parameters and survival. Ann Oncol 2008;19:883-90.

25. Witzel I, Loibl S, von Minckwitz G, et al. Monitoring serum HER2 levels during neoadjuvant trastuzumab treatment within the GeparQuattro trial. Breast Cancer Res Treat 2010;123:437-45.

26. Witzel I, Loibl S, von Minckwitz G, et al. Predictive value of HER2 serum levels in patients treated with lapatinib or trastuzumab -- a translational project in the neoadjuvant GeparQuinto trial. Br J Cancer 2012;107:956-60.

27. Ryu DW, Lee CH. Impact of Serum HER2 Levels on Survival and Its Correlation with Clinicopathological Parameters in Women with Breast Cancer. J Breast Cancer 2012;15:71-8.

28. Kong Y, Dai S, Xie X, et al. High serum HER2 extracellular domain levels: correlation with a worse disease-free survival and overall survival in primary operable breast cancer patients. J Cancer Res Clin Oncol 2012;138:275-84.

29. Thureau S, Clatot F, Laberge-Le-Couteulx S, et al. Elevated HER2 extracellular domain level in primary breast cancer with HER2 overexpression predicts early failure of adjuvant trastuzumab. Anticancer Res 2012;32:1429-33.

30. Moreno-Aspitia A, Hillman DW, Dyar SH, et al. Soluble human epidermal growth factor receptor 2 (HER2) levels in patients with HER2-positive breast cancer receiving chemotherapy with or without trastuzumab: results from North Central Cancer Treatment Group adjuvant trial N9831. Cancer 2013;119:2675-82.

31. Carney WP, Bernhardt D, Jasani B. Circulating HER2
Extracellular Domain: A Specific and Quantitative Biomarker of Prognostic Value in all Breast Cancer Patients? Biomark Cancer 2013;5:31-9.

32. Lee MH JS-Y, Kang SH, Song EJ, Park IH, Kong S-Y, et al. . The Significance of Serum HER2 Levels at Diagnosis on Intrinsic Subtype-Specific Outcome of Operable Breast Cancer Patients. PLoS ONE 2016;11:e0163370.

33. Lipton A, Ali SM, Leitzel K, et al. Elevated serum Her-2/ neu level predicts decreased response to hormone therapy in metastatic breast cancer. J Clin Oncol 2002;20:1467-72.

34. Lennon S, Barton C, Banken L, et al. Utility of serum HER2 extracellular domain assessment in clinical decision making: pooled analysis of four trials of trastuzumab in metastatic breast cancer. J Clin Oncol 2009;27:1685-93.

35. Eisenhauer EA, Therasse P, Bogaerts J, et al. New response evaluation criteria in solid tumours: revised RECIST guideline (version 1.1). Eur J Cancer 2009;45:228-47.

36. Cook GB, Neaman IE, Goldblatt JL, et al. Clinical utility of serum HER-2/neu testing on the Bayer Immuno 1 automated system in breast cancer. Anticancer Res 2001;21:1465-70.

37. Shamshirian A, Aref AR, Yip GW, et al. Diagnostic value of serum HER2 levels in breast cancer: a systematic review and meta-analysis. BMC Cancer 2020;20:1049.

38. Zheng H, Zhong A, Xie S, et al. Elevated serum HER-2 predicts poor prognosis in breast cancer and is correlated to ADAM10 expression. Cancer Med 2019;8:679-85.

39. Fehm T, Gebauer G, Jager W. Clinical utility of serial serum c-erbB-2 determinations in the follow-up of breast cancer patients. Breast Cancer Res Treat 2002;75:97-106.

40. Mazouni C, Hall A, Broglio K, et al. Kinetics of serum HER-2/neu changes in patients with HER-2positive primary breast cancer after initiation of primary chemotherapy. Cancer 2007;109:496-501.

41. Reix N, Malina C, Chenard MP, et al. A prospective study to assess the clinical utility of serum HER2 extracellular domain in breast cancer with HER2 overexpression. Breast Cancer Res Treat 2016;160:249-59.

42. Burstein HJ, Harris LN, Marcom PK, et al. Trastuzumab and vinorelbine as first-line therapy for HER2overexpressing metastatic breast cancer: multicenter phase II trial with clinical outcomes, analysis of serum tumor markers as predictive factors, and cardiac surveillance algorithm. J Clin Oncol 2003;21:2889-95.

43. Schippinger $W$, Regitnig $P$, Bauernhofer T, et al. The course of serum HER-2/neu levels as an independent prognostic factor for survival in metastatic breast cancer. Oncol Rep 2004;11:1331-6. 
44. Lipton A, Leitzel K, Ali SM, et al. Serum HER-2/neu conversion to positive at the time of disease progression in patients with breast carcinoma on hormone therapy. Cancer 2005;104:257-63.

45. Sandri MT, Johansson HA, Zorzino L, et al. Serum EGFR and serum HER-2/neu are useful predictive and prognostic markers in metastatic breast cancer patients treated with metronomic chemotherapy. Cancer 2007;110:509-17.

46. Finn RS, Gagnon R, Di Leo A, et al. Prognostic and predictive value of HER2 extracellular domain in metastatic breast cancer treated with lapatinib and paclitaxel in a randomized phase III study. J Clin Oncol 2009;27:5552-8.

Cite this article as: Zuo WJ, He M, Zheng H, Liu Y, Liu XY, Jiang YZ, Wang ZH, Lu RQ, Shao ZM. Serum HER2 levels predict treatment efficacy and prognosis in patients with HER2positive breast cancer undergoing neoadjuvant treatment. Gland Surg 2021;10(4):1300-1314. doi: 10.21037/gs-20-802
47. Köstler WJ, Schwab B, Singer CF, et al. Monitoring of serum Her-2/neu predicts response and progression-free survival to trastuzumab-based treatment in patients with metastatic breast cancer. Clin Cancer Res 2004;10:1618-24.

48. Tchou J, Lam L, Li YR, et al. Monitoring serum HER2 levels in breast cancer patients. Springerplus 2015;4:237.

49. Di Gioia D, Dresse M, Mayr D, et al. Serum HER2 supports HER2-testing in tissue at the time of primary diagnosis of breast cancer. Clin Chim Acta 2014;430:86-91.

50. Jiang YZ, Liu Y, Xiao Y, et al. Molecular subtyping and genomic profiling expand precision medicine in refractory metastatic triple-negative breast cancer: the FUTURE trial. Cell Res 2021;31:178-86. 